\title{
PATH LOSS MODELING FOR URBAN WIRLESS NETWORKS IN BAGHDAD
}

\author{
${ }^{*}$ Baseem G. Nsaif ${ }^{1}$
}

Adheed H. Sallomi ${ }^{1}$

1) Electrical Engineering Department, College of Engineering, Mustansiriyah University, Baghdad, Iraq

\begin{abstract}
An accurate propagation modeling of radio waves propagation is very important task in cellular network design as it provides the detailed useful knowledge about the wireless channel environment characteristics. Theoretical or empirical RF propagation models provide the required useful information about the signal path loss and fading to evaluate the received signal level, the coverage area, and the outage probability in specific regions. This paper aimed to develop an empirical radio wave propagation model based on observations and sets of measurement data collected from different sites through drive test. These measurements are used to determine the received signal power at some locations to create an empirical radio wave propagation model that is suitable to be appropriate in cellular network accurate design and link budget prediction at the city of Baghdad.
\end{abstract}

Keywords: Cellular mobile network design, Log-distance model, Signal fading, Empirical propagation modeling.

\section{Introduction}

The widespread demand for cellular communication service has lead mobile communication operators to use micro-cellular technology to face the extraordinary increasing in the number of subscribers. The reliable cellular mobile radio design heavily depends on accurate prediction of transmitted signal attenuation, and local field variations [1], [2].

The RF propagation model is a set of mathematical equations or algorithms through which the received signal strength or the path loss can be predicted in specific regions [2]. RF propagation models can help the radio engineer to design, and enhance radio communicating networks performance.

RF propagation model determines the required transmitted signal level for specific region coverage, plays a significant role in frequency plan setting, accurate estimation of mobile station position, base station location decision, and cell size estimation [3]. Therefore, it is very necessary to construct an accurate propagation model to estimate the cellular network parameters that affect the mobile radio network performance [4], [5].

The radio waves propagation through urban areas is very complex process, as the propagation channel is time-variant and have random characteristics. Therefore, establishing accurate path loss prediction models in cellular mobile environments represents a challenging task [3].

When line-of -sight (LOS) path is not exist or blocked by the building in urban areas, the signal arrive the receiving antenna in multipath after reflection, or diffraction [6], [7]. The transmitted radio signal will attenuate as the $\mathrm{T}$ $\mathrm{R}$ separation distance $d$ increases. The signal attenuation is proportional to $d^{\gamma}$ where $\gamma$ is the path loss exponent value [8] In addition to

*Corresponding Author: basim_gazy@yahoo.com 
signal attenuation, the propagated signal experience delay, multipath and fading [8], [9].

This paper aimed to determine the path loss exponent value in urban places at the city of Baghdad, and to develop an empirical radio propagation model that be appropriate in Baghdad. This will be carried out through the received power measurements for different distances in several cellular sites at the city. The model will be helpful for accurate base station placing, and cellular communication network design.

The research is arranged in five sections: In section 2, some selected RF propagation models are briefly described whereas section 3 , focus on the practical measurement obtained for the received signal carried out in many sites. In section 4 , the constructed empirical model is presented and simulated. The paper is concluded in the last section.

\section{Radio Path Loss Models}

The radio channel properties are mainly depend on many parameters such as operating frequency, applied antennas, and the nature of the environment through which the radio waves propagate. The mobile communication channel analysis requires to explain the radio signal propagation in free space firstly.

Free space model defines how much power of the signal is lost during the signal transmission to the receiver through the line of sight (LOS) path. The path loss $\left(P_{L}\right)$ due to power dissipation during propagation in free space where the LOS is not obstructed by any object in $\mathrm{T}-\mathrm{R}$ distance is given as:

$P_{L}(d)=20 \log _{10} d+20 \log _{10} f+20 \log _{10}\left(\frac{4 \pi}{c}\right)$

Where $d$ is the distance between the transmitting and receiving antennas, $f$ is the operating frequency, and $c$ is the speed of light $\left(3 \times 10^{8} \mathrm{~m} / \mathrm{s}\right)$.
Equation (1) shows that the path loss is proportional to the square of $\mathrm{T}-\mathrm{R}$ separation distance $(d)$. The free space propagation model is valid only for the values of $d$ that is located at the Fraunhofer zone (far-field zone) of the base station-transmitting antenna [9].

Empirical propagation models can be established for a certain environment by the data measured in the environment at the operating frequency. The most known practical mobile radio propagation model is the log-distance path loss model or so-called power exponent model.

The Log-distance path loss model indicates that the received signal power decreases logarithmically as the(T-R) separation distance increased. The average received power and the average path loss for any distance $d$ are expressed as:

$P_{r}(d)=P_{r}\left(d_{o}\right)-10 \gamma \log _{10}\left(d / d_{o}\right)$

$P_{L}(d)=P_{L}\left(d_{o}\right)+10 \gamma \log _{10}\left(d / d_{o}\right)$

Where $d_{\mathrm{o}}$ is a reference distance very close to the transmitting antenna, and located at the farfield zone of the antenna, $P_{r}(\mathrm{~d})$ is the received power at the distance $d,\left(\mathrm{~d}>\mathrm{d}_{\mathrm{o}}\right), P_{r}\left(d_{o}\right)$ is the power received at the reference distance $\left(d_{o}\right)$, $P_{L}\left(d_{o}\right)$ is the path loss at the reference distance $\left(d_{o}\right)$, and $\gamma$ is the path loss exponent value that describe the rate of path loss variation as the distance increases. The value of $\gamma$ is equal to 2 in free space, and to 4 in standard urban environments.

The terrains, buildings and other obstacles existing in the path between the transmitting cause and receiving antennas gives rise to lognormal fading or shadowing, and multipath fading. Therefore, a part of transmitted power is absorbed by obstacles resulting in random received signal due to LOS path blockage [10]. Shadowing and variable clutter along the T-R distance are not considered in Log-distance 
model. The received power measurements may not be accurate and affected by the local area clutter. The received power measurements may be different at two points with same distances from the transmitting antennas due to random clutter effects [11].

The log-normal shadowing propagation model consider signal attenuation and the random shadowing effects that causes signal level variation around its mean value. The log-normal shadowing model is given as [12]:

$P_{L}(d)=P_{L}\left(d_{o}\right)+10 \gamma \log _{10}\left(d / d_{o}\right)+X_{\sigma}$

where $X_{\sigma}$ is a Gaussian random variable with zero mean and standard deviation of $\sigma(\mathrm{dB})$. The standard deviation $\sigma$ describe the shadowing effects in random locations [13], [14].

\section{Experimental Setup and Data Collection}

The drive test routes were planned to include some accessible sites in Baghdad. The drive measurement setup consisted of a laptop connected to the spectrum analyzer. The drive test data obtained with the use of low-speed car include the site/sector number, channel frequency, and received power.

The distance between the transmitting and receiving antennas that corresponds to the propagation distance was obtained by the global positioning system (GPS) data recorded during drive test measurements.

The received signal levels at various distances away from the transmitting Base Transceiver Station (BTS) in this research were collected from more than 150 measurement points in many urban areas at different distances from base stations as a case study. The measurement procedure was repeated at many locations along the target area. The collected drive test data of the received signal power and corresponding separation distances for some sites are shown in Table1.

Table 1. Received Signal Levels with T-R Separation Distance

\begin{tabular}{|c|c|c|c|c|c|}
\hline \multicolumn{6}{|c|}{ Drive Test Measured Data } \\
\hline \multicolumn{2}{|c|}{ FIRST SITE } & \multicolumn{2}{|c|}{ SECOND SITE } & \multicolumn{2}{|c|}{ THIRD SITE } \\
\hline 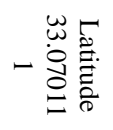 & 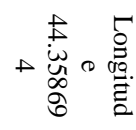 & 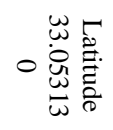 & 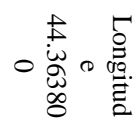 & 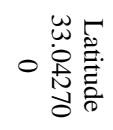 & 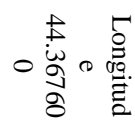 \\
\hline$\stackrel{2}{\hat{\Xi}}$ & 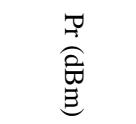 & $\stackrel{2}{\widehat{E}}$ & 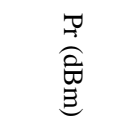 & $\stackrel{2}{\stackrel{\Xi}{E}}$ & $\underset{\overparen{\overbrace{}}}{\stackrel{7}{\tilde{E}}}$ \\
\hline 40 & -44 & 50 & -47 & 60 & -52 \\
\hline 60 & -48 & 100 & -60 & 100 & -56 \\
\hline 80 & -51 & 200 & -63 & 150 & -60 \\
\hline 100 & -56 & 300 & -67 & 200 & -64 \\
\hline 120 & -58 & 400 & -69 & 250 & -70 \\
\hline
\end{tabular}

Fig. 1 illustrates one of the cellular sites in study. The cell sites under study were all provided with sector antennas, each of $18 \mathrm{dBi}$ gain, and roof top mounted at 20-25 m heights.

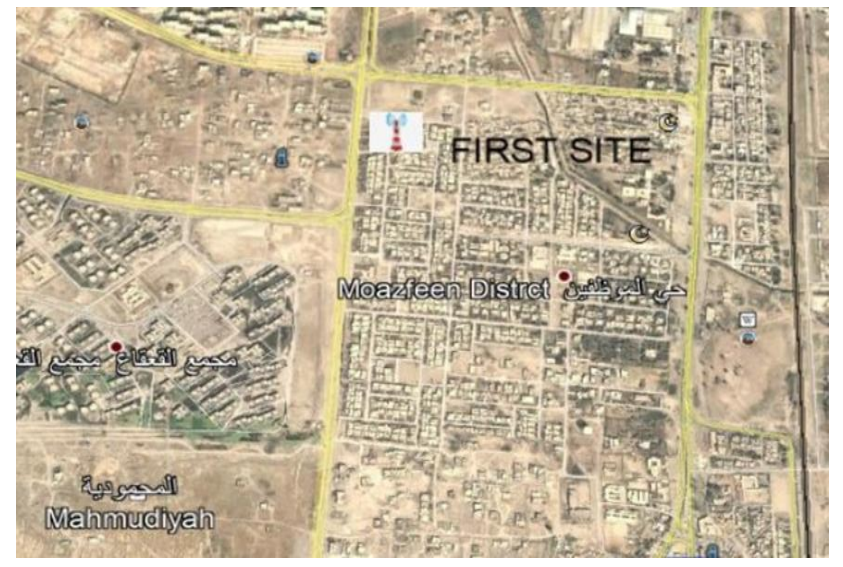

Figure 1. The Google Image of the First Site

\section{Path Loss Power Exponent Optimization}

The log-distance or power exponent path loss model can be optimized through any numerical optimization schemes that are included in the MATLAB Optimization Toolbox to calculate the power exponent value $(\gamma)$.

The Least Mean Square (LMS) algorithm is used to minimize the error (e) between the 
measured and the calculated received power levels in each test step to derive the path loss exponent value $(\gamma)$. The data shown Table 1 , can be used to find the path loss exponent value by the least squares fit, taking the minimum distance for each site as the reference distance $\mathrm{d}_{\mathrm{o}}$. For $N$ number of measurements had been carried out for each site, the Least Mean Square (LMS) error:

$$
\begin{array}{r}
F(\gamma)=\sum_{i=1}^{N}\left(e_{i}\right)^{2}=\sum_{i=1}^{N}(\text { measured power }- \\
\text { calculated power })^{2} \\
F(\gamma)=\sum_{i=1}^{N}\left(e_{i}\right)^{2}=\sum_{i=1}^{N}[\text { measured power }- \\
\left.\left(P_{r}\left(d_{o}\right)-10 \gamma \log _{10}\left(d / d_{o}\right)\right)\right]^{2}
\end{array}
$$

where $e_{i}$ is the difference between the measured and calculated values of the received signal levels, Pri is the received power as measured in $i$-th measurement at the distance $d i$.

$$
\begin{aligned}
F(\gamma)=\sum_{i=1}^{N} e_{i}^{2}= & {\left[-44-(-44-10 \gamma \log 40 / 40]^{2}+\right.} \\
& {\left[-48-(-44-10 \gamma \log 60 / 40]^{2}+\right.} \\
& {\left[-51-(-44-10 \gamma \log 80 / 40]^{2}+\right.} \\
& {\left[-56-(-44-10 \gamma \log 100 / 40]^{2}+\right.} \\
& {\left[-58-(-44-10 \gamma \log 120 / 40]^{2}\right.} \\
F(\gamma)=\sum_{i=1}^{N} e_{i}{ }^{2}= & 50.66 \gamma^{2}-285.06 \gamma+405
\end{aligned}
$$

Differentiating $\mathrm{F}(\gamma)$ relative to $\gamma$ and setting the derivative to zero yields:

$$
\begin{aligned}
& \frac{d}{d \gamma}(F(\gamma))=2 \times 50.66 \gamma-285.06=0 \\
& \gamma=\frac{285.06}{101.32}=2.8134
\end{aligned}
$$

By LMS algorithm scheme, it was found that the path loss exponent value is equal to (2.8134). The standard deviation $(\sigma)$, can be determined so that the model can be used at any location at the area of interest. The standard deviation can be calculated as:

$$
\begin{aligned}
\sigma_{d B} & =\sqrt{\frac{\sum_{i=1}^{N}(\text { power measured-power calculated })^{2}}{N}} \\
\sigma_{d B} & =\sqrt{\frac{F(\gamma)}{N}} \cong 5 d B
\end{aligned}
$$

The log-distance propagation model without considering shadowing effects can be written as:

$$
\begin{aligned}
& P_{r}(d)=P_{r}\left(d_{o}\right)-10 \gamma \log _{10}\left(d / d_{o}\right) \\
& P_{r}(d)=-44-28.134 \log _{10}(d / 40) \\
& P_{r}(d)=1.072-28.134 \log _{10}(d)
\end{aligned}
$$

The combined log-distance path loss model with shadowed sub- urban area can be modified to be:

$$
\begin{aligned}
& P_{r}(d)=1.072-28.134 \log _{10}(d)-5 d B \\
& P_{r}(d)=-3.928-28.134 \log _{10}(d)
\end{aligned}
$$

The obtained model is simulated with the use of MATLAB program as in Fig. 2.

It can be noticed that for the same distance the power received at free space is greater than that of urban area.

Fig. 3 shows the received power level variation over distance. The figure shows how the received signals decreases that as the distance increases especially in shadowed areas due to the environmental factors such as buildings and other obstacles along with the free space loss. The figure simulated the predicated model, and compares the received power at the standard urban area with path loss exponent of 4 , and free space at which the path loss exponent is equal to 2.

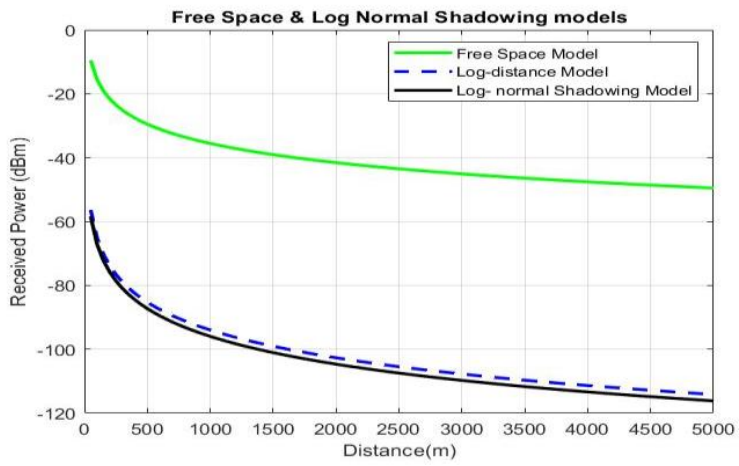

Figure 2. The received power at proposed model 


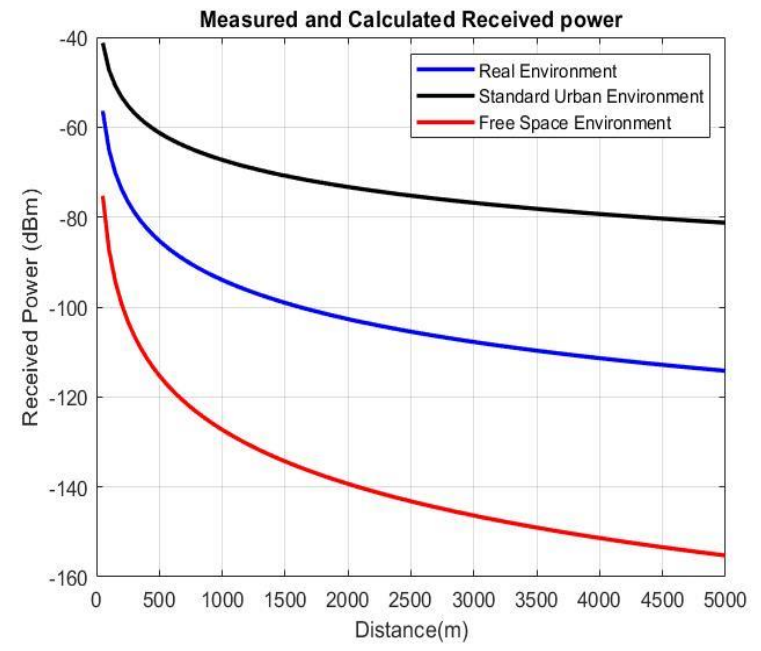

Figure 3. The received power at different models

As the obtained data exhibit nonlinear characteristics, it may be useful to use artificial neural networks in received power or path-loss prediction as an alternative method. Artificial neural networks have a potential of tuning the parametric model weight function. A neural network of measured values as an input vector, the measured output, and several hidden layers was built to obtain a deterministic path loss model. The Artificial neural model was trained using about 150 input-output pairs for $1.8 \mathrm{GHz}$. The results were plotted in Fig. 4.

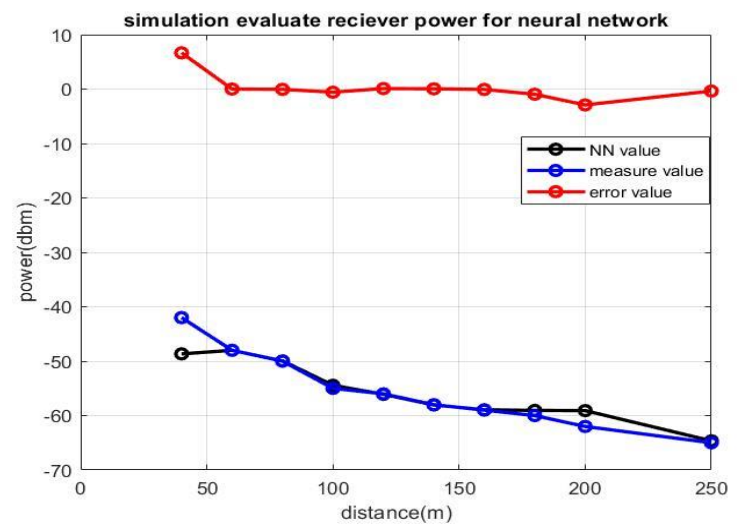

Figure 4. Neural network simulation

\section{Conclusions}

In this paper, an RF propagation model is obtained and optimized to be suitable for utilization in cellular networks operating at the city of Baghdad. This was carryout through a series of measurement by drive test measurements in some accessible sites of Zain. The path loss exponent value, was found to be equal to 2.8134 which means that the path loss is proportional to $\mathrm{d}^{2.8134}$. The calculated path loss exponent value falls within the limits of suburban area environment and shows reliable and acceptable accuracy. The standard deviation due to the obstacles existing along the communication link was calculated to be $5.0 \mathrm{~dB}$. The generated model takes the form $\mathrm{P}_{\mathrm{r}}(\mathrm{d})=1.072-28.134 \log \mathrm{d}$. It was tested to predict the received signal level at many sites.

\section{Conflict of interest}

The authors declare that the publication of this article causes no conflict of interest.

\section{References}

1. J. Zegarra, "Model development for wireless propagation in forested environments," Naval Postgraduate School Monterey United States, 2015.

2. Y. Zakaria and L. Ivanek, "Performance evaluation of channel propagation models and developed model for mobile communication," Am. J. Appl. Sci., vol. 14, no. 2, pp. 349-357, 2017.

3. R. K. Saha, "Comparative Analysis of Path Loss Models in Mobile Communications for Urban Case," no. March, pp. 1-30, 2016, doi: 10.13140/RG.2.1.3665.8324.

4. O. S. Oguejiofor, C. V Oleka, H. C. Ejiofor, and N. A. Okumbor, "Mobile Radio Link Design Using Path Loss Model," Int. J. Eng. Adv. Technol., vol. 2, pp. 421-424, 2013.

5. R. O. Aba, "Path loss prediction for gsm mobile networks for urban Region of Aba, South-East Nigeria," Int. J. Comput. Sci. Mob. Comput., vol. 3, pp. 267-281, 2014.

6. J. Isabona and $\mathrm{K}$. Obahiagbon, "RF Propagation Measurement and Modelling to Support Adept Planning of Outdoor Wireless Local Area Networks in $2.4 \mathrm{GHz}$ Band," Am. J. Eng. Res., vol. 3, no. 1, pp. 258-267, 2014.

7. F. J. Oluwole and O. Y. Olajide, "Radio 
frequency propagation mechanisms and empirical models for hilly areas," Int. J. Electr. Comput. Eng., vol. 3, no. 3, pp. 372-376, 2013.

8. T. S. Rappaport, Wireless communications: principles and practice, 2nd ed. Upper Saddle River, 2002.

9. J. S. Seybold, Introduction to $R F$ propagation. John Wiley \& Sons, 2005.

10. C. A. Oroza, Z. Zhang, T. Watteyne, and S. D. Glaser, "A machine-learning-based connectivity model for complex terrain large-scale low-power wireless deployments," IEEE Trans. Cogn. Commun. Netw., vol. 3, no. 4, pp. 576584, 2017.

11. H.-S. Jo, C. Park, E. Lee, H. K. Choi, and J. Park, "Path loss prediction based on machine learning techniques: Principal component analysis, artificial neural network, and Gaussian process," Sensors, vol. 20, no. 7, p. 1927, 2020.

12. P. Thu, Z. Tun, and A. S. Hlaing, "Modification of propagation prediction model for $2.4 \mathrm{GHz}$ indoor wireless environment," International Conference on Advances in Engineering and Technology (ICAET), 2014, pp. 360-364.

13.Z. Gao et al., "Wireless channel propagation characteristics and modeling research in rice field sensor networks," Sensors, vol. 18, no. 9, p. 3116, 2018.

14. J. Isabona and V. M. Srivastava, "A neural network based model for signal coverage propagation loss prediction in urban radio communication environment," Int. J. Appl. Eng. Res., vol. 11, no. 22, pp. $11002-$ 11008, 2016. 